\title{
Téoros
}

Revue de recherche en tourisme

\section{Téoros fait peau neuve !}

\section{Alain A. Grenier}

Volume 28, numéro 1, 2009

Tourisme polaire

URI : https://id.erudit.org/iderudit/1024831ar

DOI : https://doi.org/10.7202/1024831ar

Aller au sommaire du numéro

\section{Éditeur(s)}

Université du Québec à Montréal

\section{ISSN}

0712-8657 (imprimé)

1923-2705 (numérique)

Découvrir la revue

Citer ce document

Grenier, A. A. (2009). Téoros fait peau neuve ! Téoros, 28(1), 3-6.

https://doi.org/10.7202/1024831ar d'utilisation que vous pouvez consulter en ligne.

https://apropos.erudit.org/fr/usagers/politique-dutilisation/ 


\title{
Téoros fait peau neuve!
}

\author{
Alain A. GRENIER, Ph.D. \\ Directeur et rédacteur en chef \\ Université du Québec à Montréal \\ grenier.alain@uqam.ca
}

Ce numéro de Téoros, vous l'avez sans doute déjà constaté, se présente sous de nouvelles apparences. La revue de recherche en tourisme, Téoros, compte près de 30 années d'existence. Au cours de ces années, Téoros a connu plusieurs changements, tant dans son apparence que son contenu. Chacun de ces changements est venu marquer, en quelque sorte, la réponse de la revue aux nouveaux besoins de son lectorat, du milieu universitaire et de la recherche. Les changements que nous apportons aujourd'hui à Téoros marquent à leur façon le renouveau de la revue.

Depuis ses débuts, Téoros se consacre à l'observation, à la réflexion, au commentaire et à la diffusion des idées qui font avancer la connaissance sur l'univers du tourisme. Il y a 30 ans, l'étude du tourisme n'en était encore qu'à ses balbutiements. Le tourisme était plus perçu comme un produit commercial - souvent celui des privilégiés ou des bohèmes - que ce mode d'expression qui caractérise aujourd'hui les sociétés occidentales. La démocratisation du tourisme et la massification du phénomène, depuis les années 1960, ont amené beaucoup de critiques, tant chez les profanes que les spécialistes : acculturation, détérioration du patrimoine naturel, superficialité de l'expérience, etc. Les critiques du tourisme de masse ont contribué à l'émergence de nouvelles formes de tourisme : écologique, social, équitable, solidaire, communautaire, d'aventure, etc. Parallèlement à ces changements, le tourisme de masse continue de s'accroître, offrant une panoplie d'expériences spécialisées (tourisme de magasinage, sportif, de festival, sombre, nostalgique, etc.).

Devant la diversité des expériences touristiques qui s'offrent aux voyageurs et les pressions qu'elles exercent chacunes à leur façon sur les ressources dont elles dépendent, la gestion du tourisme nourrit aujourd'hui de nombreux débats. On multiplie les modèles de gestion sans pourtant parvenir à éliminer complètement les failles, les iniquités et les autres impacts négatifs qui accompagnent encore souvent le développement des produits et services touristiques. Pourquoi? D’une part, la connaissance des expressions du tourisme est souvent superficielle, inappropriée ou incomplète. Une gestion adéquate et efficace nécessite une meilleure compréhension de l'expérience touristique et de ses acteurs (gestionnaires, entrepreneurs, clients et communautés hôtes). Une gestion adéquate sous-entend du même coup le besoin d'approfondir la compréhension des concepts qui constituent l'essence même des produits et services conçus pour les touristes.

Les débats d'idées qui animent l'évolution et le développement des connaissances sur le tourisme nécessitent plus que jamais le développement d'arguments structurés et organisés, fondés sur des processus méthodologiques clairement exprimés. Téoros n'est pas en reste. Suivant le désir d'offrir à son lectorat un contenu toujours plus approfondi et de contribuer à l'édifice de la connaissance sur le tourisme, Téoros entame un nouveau virage. Avec ce numéro, Téoros devient une revue entièrement scientifique. Ce passage signifie simplement que tous les articles désormais présentés dans les pages de Téoros (essais, articles de réflexion, comptes-rendus d'étude), présenteront une démarche méthodologique - tant dans l'organisation des idées que la collecte des données et leur analyse - assujettie à l'approbation des pairs.

Pour réaliser ce virage, Téoros s'est dotée d'un comité scientifique (voir page générique) auquel s'ajouteront, selon les thématiques présentées dans ses pages, d'autres experts issus de plusieurs sphères d'étude du tourisme, répartis en autant d'universités et de centres de recherche à travers le monde. La responsabilité de ce comité consiste à assurer aux lecteurs de Téoros des écrits à l'avant-garde des développements dans la recherche sur le tourisme.

Pour marquer la transition, Téoros fait peau neuve et adopte une nouvelle mise en page plus adaptée à son contenu. En outre, la couverture accordera dorénavant 
davantage d'espace au contenu de l'édition ainsi qu'aux collaborateurs. L'intérieur de la revue a aussi été remanié afin d'offrir une expérience de lecture rehaussée. Le tourisme étant un sujet souvent très visuel, nous souhaitons continuer à illustrer les articles de photographies, de cartes et autres graphiques pouvant aider à une meilleure compréhension des idées proposées et développées par les auteurs.

Comme par le passé, Téoros consacrera chacun de ses numéros à une ou deux thématiques spécifiques (conceptualisation, expérience, gestion, tendances, etc.). Ces dossiers seront accompagnés d'articles hors thème, au gré des collaborations proposées. Nous vous invitons donc à contribuer en nous faisant parvenir vos textes pour publication.

S'ajouteront à ces dossiers deux nouvelles chroniques. La première, intitulée "Dans les coulisses de la science", proposera dans chaque numéro un entretien avec une personnalité marquante du domaine de la recherche en tourisme, du Québec et de l'étranger. Pour lancer la série, nous rencontrons le professeur Achille Braquelaire, mathématicien français et vice-président de l'Université Bordeaux I, qui utilise la bande dessinée - médium plutôt étranger au monde des sciences - pour offrir une image du monde scientifique et des environnements naturels sur lesquels il travaille avec ses collaborateurs. Téoros consacrera aussi une page à la recension d'ouvrages universitaires sélectionnés en relation avec les thématiques développées dans ses dossiers.

Les nouvelles exigences de production de Téoros nécessitent de ramener le nombre de numéros à deux annuellement - sans pénaliser les présents abonnés.

Je profite de l'occasion pour saluer l'excellent travail de mon prédécesseur, le professeur Bruno Sarrasin de l'ESG-UQAM, et de son adjointe, Barbara Julien. Les six années du professeur Sarrasin à la direction de la revue nous ont valu la présentation de dossiers de qualité et la construction d'une équipe de direction solide que je remercie également pour son travail et son soutien. Téoros poursuit sur cette envolée avec une équipe renouvelée en la personne de Mathieu Dormaels, adjoint à la rédaction, et de Marie-Claude Béland et Alain Bolduc de B Graphistes. Je salue aussi l'apport inestimable de Véronique Israël, du Département d'études urbaines et touristiques de l'ESG, à l'administration des finances de la revue.

En occupant le créneau de la recherche, Téoros devient la seule revue scientifique en tourisme de langue française. Elle constitue ainsi le véhicule de diffusion des travaux de recherche en tourisme. Plus que jamais, Téoros compte sur la participation de ses lecteurs et de ses auteurs pour contribuer non seulement à la diffusion de la connaissance sur le tourisme, mais aussi à son édifice. Que vous soyez voyagiste, consultant, gestionnaire, étudiant ou chercheur, nous espérons que vous trouverez dans Téoros l'occasion d'une lecture enrichie.

\section{Numéro polaire}

Le passage de Téoros à sa nouvelle formule scientifique correspond à la fin de l'Année polaire internationale (mars 2007-2009 - une année plus longue pour marquer le cycle complet en Arctique et en Antarctique). Il convenait donc à juste titre d'inscrire Téoros au chapitre de la contribution à la recherche en tourisme polaire.

La collection de textes que vous trouverez dans nos pages offre un échantillon représentatif des diverses thématiques abordées en tourisme polaire. Ces textes représentent les enjeux les plus importants, tant pour l'Arctique que l'Antarctique : le rôle de la mythologie polaire dans la construction culturelle des attraits, l'activité touristique comme moyen d'appropriation du territoire, les enjeux identitaires du développement touristique en milieu inuit, le cadre juridique du tourisme en Antarctique et l'impact des changements climatiques sur le tourisme polaire.

En guise d'introduction à la thématique de ce numéro, je propose un texte de réflexion sur le concept de tourisme polaire. Si le tourisme polaire n'est pas nouveau (voir le texte de Denis Jallat dans ce numéro), personne n'a jusqu'à présent tenté d'en circonscrire le concept. À défaut d'en saisir l'essence, les appellations se multiplient : tourisme polaire, nordique, hivernal, antarctique, alpin, etc. Le tourisme polaire dépasse$\mathrm{t}$-il le seul cadre des croisières et des cercles polaires?

Les croisières en milieux polaires occupent, il est vrai, une part importante - voire disproportionnée - de la recherche en tourisme polaire. Ce numéro de Téoros n'y échappe pas. La majorité des textes s'y consacrent, en tout ou en partie. Les activités terrestres, pourtant plus importantes, tant en diversité qu'en termes de participants, ne semblent pas attirer autant l'attention des chercheurs. Or, les besoins y sont tout aussi importants, souvent encore davantage. Il faut dire que l'attrait des régions polaires mène souvent à une quête personnelle des pôles. Le tourisme polaire - il n'y a pas de consensus sur une façon de le définir - est intimement lié à la géographie. Inévitablement, l'étude des pôles se mesure en longitude et (surtout) en latitude. La distinction, moteur du tourisme (Boyer, 1995), s'applique non seulement aux touristes, mais à ceux et à celles qui les étudient. Au-delà des considérations scientifiques, la poursuite d'objectifs de recherche et de carrière stimule sans doute les chercheurs à explorer un maximum de lieux qu'elles ou ils collectionnent à travers leurs études. La popularité de la croisière polaire comme sujet d'étude par les jeunes chercheurs est donc évidente. La navigation demeure l'unique moyen d'accéder aux terres les plus extrêmes des deux hémisphères de la planète. Leur caractère relativement peu connu, les coûts liés à leur accès et les difficultés engendrées par la navigation sur les mers polaires suffisent à faire miroiter les promesses d'aventure, dont rêvent aussi les chercheurs.

Le tourisme polaire propose cependant un environnement d'étude particulièrement approprié pour certains types d'études (sociologiques, notamment), puisqu'il octroie aux chercheurs un milieu fermé où étudier les comportements des voyageurs. La croisière permet aussi d'étudier les modèles de gestion des voyagistes, tant en regard de la faune et de la flore des sites visités que des contacts avec les autochtones (dans l'Arctique) et des scientifiques (dans les stations de recherche en Antarctique). L'impact des changements climatiques, spécialement ressenti dans les régions polaires (particulièrement en Arctique), multiplie les problématiques 
associées à la demande croissante pour ce type de voyage, au développement d'infrastructures qu'il nécessite et à la volonté (ou à la résistance parfois) des communautés hôtes de contribuer à ce développement.

Denis JALLAT, maître de conférences à l'Université de Strasbourg II où il enseigne l'histoire des sports et des loisirs, analyse les débuts du tourisme polaire pour la clientèle française dans les années 1930, autour de l'archipel norvégien de Svalbard. Il expose le contexte particulier de l'époque et comment celui-ci favorisa le développement des croisières en milieu polaire.

Nous restons au Svalbard où Ricardo ROURA, doctorant du Centre arctique de l'Université de Groningen (PaysBas), nous propose l'étude d'un site du patrimoine polaire au Svalbard. Il s'intéresse aux formes narratives employées pour transformer un site patrimonial en attraction touristique. Son étude de cas est d'autant plus intéressante que l'attraction n'y est ni plus ni moins que le mât ayant servi de point d'attache du Norge, le dirigeable lancé le 12 mai 1926 par l'équipe de Roald Amundsen pour survoler le pôle Nord. Le vestige est situé à Ny-Ålesund, la communauté la plus septentrionale de la planète $\left(78^{\circ} 56^{\prime} \mathrm{N}\right)$. À l'aide d'observations menées sur le terrain, Ricardo Roura décortique le rôle du tourisme dans la sacralisation d'un lieu.

Cette sacralisation par les touristes n'est d'ailleurs pas sans rappeler que le tourisme est un mode de consommation de l'espace (Urry, 1995). Dans cette perspective, Sophie DUPRÉ, stagiaire postdoctorale à l'Université du Québec à Rimouski, recense les principales attractions de ce territoire, lui aussi fréquenté par les navires de croisière.

Nous profiterons de ce détour en sol canadien pour faire escale à Puvirnituq, au Nunavik. Véronique ANTOMARCHI, professeure agrégée, poursuit dans la même veine que le texte précédent et approfondit les problématiques liées au développement du tourisme dans les communautés autochtones de l'Arctique canadien : lutte à la pauvreté, valorisation du patrimoine culturel, positionnement politique dans l'administration des territoires, etc. Le portrait qu'elle dresse, s'il est très positif, montre aussi l'ampleur du travail nécessaire avant d'en arriver à une activité touristique stable et fiable.

Outre le respect des cultures autochtones, l'autre grand enjeu du tourisme polaire réside dans la protection des espaces visités. Le défi est d'autant plus grand que le tourisme polaire se produit généralement durant le court été, pendant la période de renouvellement et de croissance des végétaux et de la faune qui habitent le territoire. Les bons principes peuvent-ils suffire à encadrer le développement durable de l'industrie touristique? Comment superviser une industrie dont les activités échappent encore souvent aux cadres juridiques des lieux visités? À partir d'une étude de cas sur le cadre juridique des activités touristiques en Antarctique, Anne CHOQUET, docteure en droit et enseignante à l'Université de Bretagne occidentale (Brest, France), tente de répondre à ces questions. Sa réflexion part du principe que même si «le seul moyen de ne pas polluer la région, c'est de ne pas y aller", le tourisme polaire et les activités non gouvernementales «constituent des activités légitimes de la région». Elle souligne la lourdeur administrative du Traité sur l'Antarctique et déplore que, «la plupart du temps, seules des résolutions sont adoptées et non des mesures». Voilà bien l'essence de la difficulté de gérer l'activité humaine sur des territoires trop vastes, sans supervision.

Notre étude du tourisme polaire se referme sur une question diamétralement différente : celle de l'impact des changements climatiques sur la valorisation commerciale (marketing) des régions nordiques. C. Michael HALL, professeur de l'Université de Canterbury (Nouvelle-Zélande), actuellement en poste à l'Université d'Oulu (Finlande), questionne les conséquences du réchauffement climatique sur le tourisme de Noël en Laponie et sur sa plus grande attraction : le père Noël. Si la chose semble amusante, $a$ priori, il n'en est rien. Rovaniemi, capitale de la Laponie finlandaise, est l'une des rares destinations où le tourisme polaire se produit principalement en hiver, avec une pointe importante autour de la période des fêtes. Le succès de cette industrie de masse repose sur la mythologie de Noël, incarnée par la présence des rennes, des forêts laponnes figées dans la neige et du père Noël. Depuis quelques années, un réchauffement anormalement élevé des températures pendant la période d'achalandage a forcé l'industrie à adopter des stratégies pour contrecarrer l'absence de neige. Parions que les questions soulevées par C. Michael Hall trouveront un auditoire chez les voyagistes des autres destinations nordiques, ces dernières craignant elles aussi la fonte prématurée de la neige dont leur tourisme dépend.

Il faut souligner, au passage, la participation exceptionnelle du professeur Hall à cette revue. La recherche scientifique, en tourisme, est particulièrement abondante en anglais. Les chercheurs francophones, comme leurs collègues d'autres cultures (parlez-en aux Finlandais), publient régulièrement en anglais pour s'inscrire à la discussion internationale (l'inverse est moins vrai) (à ce propos, nous souhaitons un franc succès à l'équipe d'Ara, revue de recherche scientifique de tourisme en espagnol). En voulant s'imposer comme l'outil scientifique de discussion du tourisme en français, le travail de Téoros est pionnier. La participation du professeur Hall à ce numéro (il a choisi d'être publié en français) est un exemple intéressant des bénéfices que peut engendrer la transgression des barrières linguistiques pour favoriser le partage des connaissances. Souhaitons que d'autres grands noms du tourisme emboîtent le pas et favorisent la diffusion de la connaissance dans d'autres langues.

Le Nord circumpolaire est en transition. Les thématiques étudiées dans ce numéro en témoignent. Elles soulèvent toutes des questions d'actualité. La crise environnementale, la soif du «Sud» pour les ressources du Nord, l'émancipation des peuples autochtones, la sensibilisation à l'environnement des urbains, le besoin (nostalgique) d'authenticité des touristes en provenance du «Sud», le goût de modernité des résidents du Nord, le besoin des aînés de préserver leur culture : voilà quelques-unes des tensions qui s'articulent autour du tourisme. Les régions polaires sont aujourd'hui à la croisée des chemins. Certains comptent sur le tourisme pour stimuler 
leur économie et offrir une source de revenus aux jeunes à la recherche de travail. D'autres perçoivent le tourisme comme une source de contamination externe. Certaines communautés désirent le tourisme; d'autres (moins nombreuses toutefois) le rejette. Le Nord circumpolaire compte à peine 4 millions de personnes réparties sur $8 \%$ du territoire de notre planète (ADHR, 2004 : 17-19). Leurs réalités sont diverses; parfois aux antipodes. Il n'existe pas un, mais plusieurs «Nords». Les réponses aux problématiques socioéconomiques et culturelles de l'Arctique (certaines présentées dans le présent ouvrage) nécessitent par conséquent des modèles adaptés à chacune de ces réalités.

Ce numéro de Téoros contribue, à sa façon, à la discussion. L'une de ses particularités est de permettre à des chercheurs de la relève de côtoyer, dans ses pages, des chercheurs établis. Cette coopération entre chercheurs, mais aussi avec les autres acteurs du tourisme polaire et notre lectorat, se poursuit bien au-delà, par le biais du Réseau international de recherche en tourisme polaire (www.polartourismnetwork.uqam.ca), créé au DEUT (UQAM) en septembre 2007 en réponse à la demande des chercheurs. Fort de son premier colloque tenu à Kangiqsujuaq au Nunavik en août 2008, les membres du Réseau polaire ont choisi de se retrouver en Suède, en 2010, pour poursuivre les échanges.

D’ici là, bonne lecture!

Bibliographie

AHDR - Arctic Human Development Report (2004), Stefansson Arctic

Institute, Akureyri, Islande.

BOYER, Marc (1995) «L'invention de distinction, moteur du tourisme?

Hier et aujourd'hui», Téoros, vol. 14, n 2, p. 45-47.

URRY, John (1995), Consuming Places, Routledge, Londres.

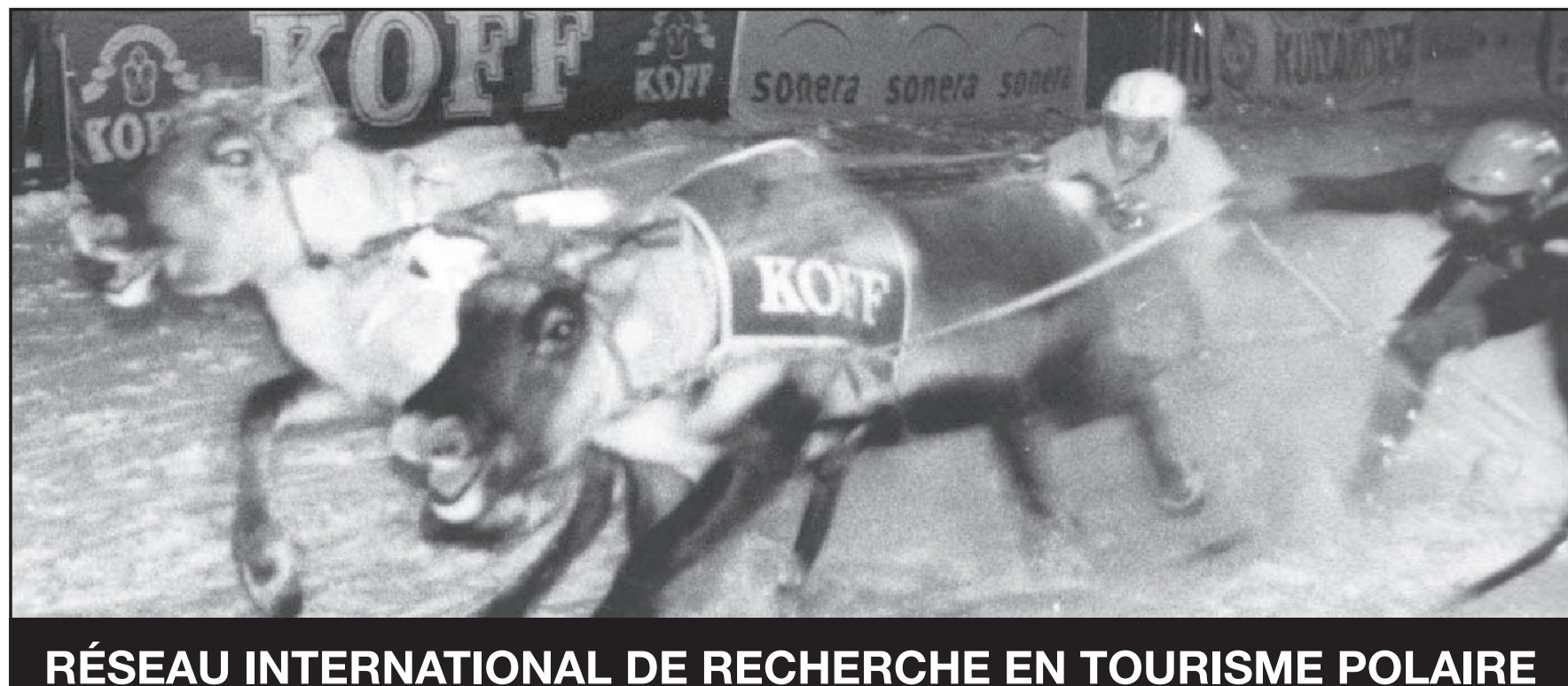

- regroupe des personnes (chercheurs, consultants, voyagistes, organisations gouvernementales, membres des communautés où se produit le tourisme polaire) qui partagent un intérêt commun pour la recherche et une meilleure compréhension du tourisme relatif aux régions polaires;

- génère et diffuse la connaissance et les points de vue sur le tourisme polaire;

- encourage le soutient, le partage des ressources, le développement de la coopération internationale et les liens entre les membres.

Soyez de la partie, devenez membre aujourd'hui! www.polartourismnetwork.uqam.ca Inscription gratuite. Renseignez-vous à: polartourism@uqam.ca 